\title{
Scham, Schuld und Peinlichkeit
}

\author{
Über die Neudefinition von Schamgrenzen
}

Klaas Huizing

\section{Möbelhaus, später}

") Waren Sie nicht mal im Fernsehen?

Ertappt, denke ich. Wie ein Stromstoß pulst das Wort durch meine Hirnlappen. >In irgend so einer Talkshow ....

Ertappt. Ich halte eine Lasche mit Ledermustern in meiner linken Hand und wollte gerade zurück zu einem Kunden mit schwachsinnigen Extrawünschen, deren Komplexität mich völlig in Beschlag nimmt und deren Absurdität mein Resthirn mit komplizierten Herstellerverzeichnissen und Materialeigenschaften blockiert.

Währenddessen stellt sich mir das fragende Ehepaar einfach in den Weg. Ohne Begrüßung, ohne Höflichkeitsabstand. [...]

Bis vor wenigen Monaten habe ich [...] für die wichtigsten Magazine und Zeitungen geschrieben, habe dafür als sogenannte Edelfeder fast alle wichtigen deutschen Journalistenpreise gewonnen; notorisch unrasiert und geistreich. Jetzt allerdings trage ich eine seriöse Krawatte, eine Bundfaltenhose und bin selbstverständlich glatt rasiert. Dabei habe ich inzwischen nichts Schlimmes verbrochen. Ich bemühe mich lediglich darum, dass mein Sohn regelmäßig essen kann. Weil die ganze Edelfederei inzwischen kein Geld mehr bringt, genausowenig wie das Albern mit Hollywood-Größen oder Partybesuche mit ShowSternchen. Und gleichzeitig ist mir das alles unsagbar peinlich. An Hollywood-Maßstäben gemessen bin ich nämlich grandios gescheitert. [...]

Jugendsünden ...<, sage ich lächelnd und hebe die Ledermuster vor mein Gesicht. «1

Dieses Langzitat entstammt, so der auf den realen Erfahrungsschatz pochende Untertitel, aus einem Tatsachenroman, geschrieben von

1 Robert Kisch, Möbelhaus. Ein Tatsachenroman, München 2015, $5 \mathrm{f}$. 
einem seriösen, weil preisgekrönten Journalisten, der in der Finanzund Zeitungskrise von den Chefredakteuren plötzlich vergessen wird, eine Amnesie mit Folgen; der Protagonist landet schließlich als Möbelverkäufer auf schäbiger Provisionsbasis in der Provinz. Der Autor wählt, um die öffentliche Scham zu vermeiden, in Anspielung auf den rasenden Reporter Egon Erwin Kisch dessen Nachnamen als Pseudonym, als Vornamen entscheidet er sich für Robert, den fliegenden Robert, eine hübsche Volte für einen, der aus dem journalistischen Betrieb rausgeflogen und unsanft gelandet ist. Und dieser Autor rast und wütet, versteckt sein Gesicht nicht nur hinter Ledermustern, sondern hinter dem riesigen Berg von Schrecklichkeiten in hochauflösenden Bildern als Langstreckenläufer in der furnierten, mit Paneelen ausgekleideten Hölle. Vom kategorischen Imperativ Kants und der Personenwürde haben weder Kunden noch Kollegen jemals etwas gehört: »Für die meisten Kunden bist du als Verkäufer entweder Personal, also kein Mensch, sondern ein Neutrum [...] oder ein abgezockter, mit allen Wassern gewaschener Betrüger. Also auch kein Mensch. $\ll^{2}$ Die ökonomische Mobilmachung kennt nur Opfer. Selbst bekennende anthropologische Optimisten bekommen spätestens auf halber Lesestrecke dieses Romans merklich Zweifel an der eigenen Optik.

Dem Protagonisten Robert Kisch ist sein unbequemes Leben in der Möbelhölle peinlich. Dafür schämen würde er sich mutmaßlich erst, wenn er plötzlich auf einen Freund oder Menschen träfe, der ihm extrem wichtig ist und der ihm durch seinen vielleicht sogar mitfühlenden Blick sein berufliches Versagen zurückspiegelt. Dann wären auch die Korridore des Versteckens verbarrikadiert. Die elementare Grundstruktur der Schamsituation lautet: Ich schäme mich für etwas, dem ich einen Wert beimesse, vor Dritten, die mir wichtig sind; oder wie Bernard Williams sagt: "Die Grunderfahrung der Scham besteht darin, dass ich von den falschen Leuten in einer falschen und unangenehmen Lage auf unangenehme Weise gesehen werde. ${ }^{3}$ In diesem Fall wäre die finale Ruinierung des Öffentlichkeitsbildes von Robert Kisch unausweichlich.

Offenbar gibt es eine Abstufung zwischen Scham und Peinlichkeit. Um diese Abstufung genauer zu beschreiben, hilft ein kurzer Besuch beim Doyen der neueren Gefühlstheorie, beim Kieler Hermann Schmitz.

\footnotetext{
2 Ebd., 178.

3 Bernard Williams, Scham, Schuld und Notwendigkeit. Eine Wiederbelebung antiker Begriffe der Moral, Berlin 2000, 91.
} 


\section{Kleine Phänomenologie der Scham, Schuld und Peinlichkeit}

In der Philosophie trägt vor allem die lange von Kollegen absichtsvoll und schnöde übersehene Leibphilosophie von Hermann Schmitz, entwickelt in seinem milde ausufernden System der Philosophie seit den späten 60er Jahren, dazu bei, Gefühle, verstanden als "räumlich ergossene Atmosphären und leiblich ergreifende Mächte", als zentralen Gegenstand der Philosophie zu erkunden. ${ }^{4}$ Längst zählt diese Leibphilosophie von Hermann Schmitz, die die - wie er markig sagt - "Menschspaltung $\aleph^{5}$ in Körper und Seele rückgängig machen will, zu den neuen Klassikern der Debatte.

Scham zählt für Schmitz neben Zorn als Reaktion auf eine verletzte Ehre zu den zentralen moralischen Gefühlsmächten. Er deutet das Gefühl der Scham, verstanden als bedrängende Atmosphäre, als ein Gefühl, das in der Moralität deshalb eine zentrale Autorität besitzt, weil alle Versuche sich davon zu dispensieren, scheitern.

"Die Vernunft (die personale Emanzipation) ist für die Moral erforderlich, um die Autorität von Gefühlen kritisch auf die Probe zu stellen und daran, dass sie sich in der Probe geschlagen geben muss, den unbedingten Ernst moralischer Gefühle zu erweisen. [...] [J]eder muss sich selbst, wenigstens in der Moral und der Religion, Rechenschaft davon geben, welche Normen mit absolutem Ernst verbindlich für ihn gelten. Wer sich diese Selbstprüfung erspart, indem er vermeintlich ewige Normen bloß abliest und auf sich selbst und andere anwendet, betrügt sich selbst über seine Verantwortung. ${ }^{6}$

Typisch für die Herangehensweise von Schmitz: Weil alles auf die leiblich-affektive Betroffenheit abgestellt wird, ist auch das Gefühl der Scham vor Ort der eigenen Leiblichkeit zu prüfen. Als Leib existierend ist, so die Pointe, jeder Mensch schon immer resonant

\footnotetext{
4 Hermann Schmitz, Atmosphären, Freiburg i.Br. 2014, 30; ders.: System der Philosophie: Der Leib; Bd. 2.1, Bonn ${ }^{2} 1982$; ders.: Der Leib, der Raum und die Gefühle, Stuttgart 1998. Dazu: Kerstin Andermann/Hermann Schmitz - Leiblichkeit als kommunikatives Selbst- und Weltverhältnis, in: Leiblichkeit, hg. v. Emmanuel Alloa u.a., Tübingen 2012,130-145.

5 Schmitz, Atmosphären, 8. Anstrengend und auch oft mühsam ist die Arbeit mit Schmitz, weil Gefühle für ihn nicht im Inneren nisten. Seine Verwendung des Wortes widerstreitet dem heute üblichen Gebrauch. Häufig gleicht die Verwendung von Gefühl eher dem religiösen Geistbegriff. Nicht zufällig ist Schmitz ekstatisch begeistert von frühchristlichen Texten, namentlich den Texten von Paulus.

6 Hermann Schmitz, Das Reich der Normen, Freiburg i.Br. 2012, 141, 144.
} 
mit seiner Umgebung und damit mit Anderen verbunden. Im Blick des Anderen kann sich die Gefühlsmacht der Scham verdichten, oder wie Schmitz sagt: inkarnieren, und mit unbedingtem Ernst den Menschen heimsuchen. Hochgradig moralisch ist diese Erfahrung, weil Scham im Unterschied zur Schuld auf die ganze Persönlichkeit und nicht auf einzelne Handlungen abzielt. ${ }^{7}$

Sehr nachdrücklich hat auch der amerikanische Philosoph Bernard Williams darauf aufmerksam gemacht, dass im Unterschied zur Schuld die Scham für moralische Selbstkritik entscheidend ist, weil sich die Scham, darin von der Schuld unterschieden, nicht leichthändig wieder gutmachen lässt.

"Die Schuld vermag Aufmerksamkeit auf die zu lenken, denen Unrecht oder Schaden zugefügt wurde, und sie verlangen Wiedergutmachung im Namen dessen, was diesen Menschen passiert ist. Aber sie versetzt uns als solche nicht in die Lage, unser Verhältnis zu derartigen Ereignissen zu verstehen, und sie hilft auch nicht dabei, das Selbst, das diese Dinge getan hat, oder die Welt, in der es leben muss, neu zu schaffen. Das kann nur die Scham, da sie eine Vorstellung davon beinhaltet, wer ich bin und in welchem Verhältnis ich zu anderen stehe. ${ }^{8}$

René Majer pflichtet in einer konzisen Studie Williams bei, modifiziert aber leicht die Pointe:

"Schuld ist nicht ausschließlich auf das Opfer bezogen. Es ist durchaus eine selbstbezogene Einstellung: Ich darf mich nicht beklagen, wenn andere wütend, erbost und einfach nur verletzt sind.` Schuld konfrontiert den Täter mit dem Groll, den seine Handlung und seine Motive verdienen. Aber Schuld sieht dabei tatsächlich vom Charakter ab. Bei Schuld geht es nicht um die Frage, was etwa Grausamkeit über die eigene Person aussagt, sondern worauf man sich angesichts der eigenen Grausamkeit gefasst machen sollte. Schuld ist selbstbezogen, ohne charakterbezogen zu sein. Gerade deswegen kann Schuld den Blick einer Person auf sich selber verstellen und die Selbstverbesserung blockieren. [...] Die Kritiker des Schuldgefühls wie Williams haben Recht, wenn sie argumentieren, dass sich auf Schuldgefühlen alleine kein moralisches Selbstverständnis aufbauen lässt. «"

\footnotetext{
7 Eine Ethik, die sich nur am Handlungsbegriff orientiert, ist deshalb eindeutig zu eng konzipiert.

8 Williams, Scham, Schuld und Notwendigkeit, 110.

9 René Majer, Scham, Schuld und Anerkennung, Berlin 2013,115. Einen möglichen Einwurf räumt Majer aus: „Man könnte hier einwenden, dass es grundsätzlich auch
} 
Majer zeigt in seiner Dissertation, wie stark Anerkennung Schamund Schuldprozesse steuert: "Zustimmung und Wohlwollen, Gegenseitigkeit und sozialisierte Selbstbezogenheit sind die wichtigsten Merkmale von Anerkennungsbeziehungen. [...] Scham- und Schuldgefuihle [sind, K.H.] immer auch Ausdruck von Anerkennungskonflikten. ${ }^{10}$

Wenn Schmitz Scham von Peinlichkeit abgrenzt, dann deshalb, weil Peinlichkeit nicht mit unbedingtem Ernst ergreift. Klar wird der Zusammenhang, wenn man auf das eigenleibliche Spüren rekurriert: In der Peinlichkeit ist die zentripetale Bewegungsrichtung abgepuffert, man zieht den Kopf nur etwas ein, wendet ihn leicht $a b$, die Augen werden zusammengekniffen, hebt vielleicht abwehrend sogar die Hände. Der `Bewußthaber, so Schmitz, ist nur noch speinlich berührtı, die Situation ist ihm aber nicht mehr sentsetzlich peinlich ${ }^{11}$ wie in der Scham; er will deshalb nicht mehr vor brennender Scham im Erdboden versinken. Je weiter der Radius der Peinlichkeit sich erstreckt, je verdünnter wird die Peinlichkeit. Im Rekurs auf die Leiblichkeit kann man Peinlichkeit und Scham prägnant voneinander abgrenzen, sofern man »in der Peinlichkeit noch den Blickkontakt mit anderen suchen kann, aber nicht muß,

möglich ist, sich schuldig für das zu fühlen, was man ist. Man kann sich schuldig für seine Homosexualität fühlen, weil man [...] fürchten kann, seine Eltern zu enttäuschen. Williams geht stillschweigend davon aus, dass solche Schuldgefühle, wenn sie auch manchmal vorkommen, dennoch niemals angemessen sind und auch nicht kultiviert werden sollten: Niemand schuldet irgendeiner anderen Person, etwas zu sein.« Ebd., 108. Majer übersieht aber, dass Scham Warnsignal für einen möglichen Übertritt in die Schuld ist. Sein Lehrer Peter Bieri übernimmt viele der Einsichten in Peter Bieri, Eine Art zu leben: Über die Vielfalt menschlicher Würde, München 2013, 157-212. Ob Scham-Sanktionen einen Platz auch im Strafrecht haben sollten, ist umstritten, Martha C. Nussbaum hat sich dagegen ausgesprochen. Martha C. Nussbaum, Hiding from Humanity. Disgust, Shame, and the Law, Princeton 2004.

${ }^{10}$ René Majer, Scham, Schuld und Anerkennung, 4. Majer betont zu Recht, es gebe Schuld, die schlechterdings nicht zu vergelten sei. »Wo aber Wiedergutmachung unmöglich ist, erübrigt sich auch die Pflicht dazu. Es mag ungewohnt klingen, aber wer etwas anrichtet, das nur schlimm genug ist, hat eine Sorge weniger." Ebd., 9.

${ }^{11}$ Hermann Schmitz, Der Leib, Berlin 2011, 90. Zur Peinlichkeit vergleiche Norbert Elias, Über den Prozeß der Zivilisation. Soziogenetische und psychogenetische Untersuchungen. Zweiter Band: Wandlungen der Gesellschaft. Entwurf zu einer Theorie der Zivilisation (1939), Frankfurt a.M. 1997, 414: »Peinlichkeitsgefühle sind Unlusterregungen oder Ängste, die auftreten, wenn ein anderes Wesen die durch das Über-Ich repräsentierte Verbotsskala der Gesellschaft zu durchbrechen droht oder durchbricht." 
und [zugesteht, K.H.] daß der Impuls, aus der Situation zu fliehen, erheblich schwächer ist als in der Scham. «12

Im Schmitz-Sound hört sich das so an:

"Gelegentlich kommt es vor, dass jemand sich beschämend benimmt, sich aber selbst nicht schämt, während die Anwesenden und Angehörigen peinlich berührt sind. Dann erweist sich die Scham als Atmosphäre, die sich von dem sich nicht Schämenden oder, in anderen Fällen, sich auch Schämenden in den Bereich merklicher Anwesenheit oder auch darüber hinaus erstreckt, bis an einen Rand, wo sie nur noch als Peinlichkeit gespürt wird, von der man noch berührt, aber nicht mehr durch und durch ergriffen ist. Der peinlich Berührte will nicht mehr wie der von katastrophaler Scham Ergriffene im Boden versinken, aber er würde lieber weg sein; er senkt nicht den Blick, kneift aber die Augen etwas zu, um nicht zu genau hinzusehen. Diese Scham ist eine Atmosphäre, die in ihrem Verdichtungsbereich vernichtend, aber zum Rand hin verdünnt ist; in beiden Fällen ist es dieselbe Scham, die im ersten Fall sagen lässt 'Es ist mir entsetzlich peinlich, dass ...<, im zweiten Fall >Ich bin peinlich berührt von ..... $\ll^{13}$

In Situationen der Peinlichkeit besteht auch für denjenigen, der Auslöser der Peinlichkeit ist, kein vergleichbarer Druck wie in der Scham, die Scham in die Schuld zu verschieben, um aus der Passivität der Schamsituation in die Aktivität der Schuldsituation zu wechseln. Offenbar lässt sich mit Schuld häufig besser leben als mit Scham. Wer die Scham nicht in die Schuld verschiebt, der ist gezwungen, seinen eigenen Charakter umzuformen, lebt künftig mit vorweggenommener Scham, um vergleichbar kritische Situationen zu vermeiden. Sich schämen oder über sein eigenes Verhalten Peinlichkeit empfinden, kann allerdings nur derjenige, der die Normen - hier in einem sehr weiten Sinn verstanden - teilt, die bei einem Verstoß zu einer entsprechenden Reaktion durch Dritte führt. In spätmodernen multikulturellen Gesellschaften dient die Scham wie in frühmodernen Gesellschaften ${ }^{14}$ zur Angleichung von Verhaltensstandards; sie

12 Hilge Landweer, Scham und Macht. Phänomenologische Untersuchungen zur Sozialität eines Gefühls, Tübingen 1999, 165.

${ }^{13}$ Hermann Schmitz, Der Leib, Berlin 2011, 90.

${ }^{14}$ Im Rekurs auf das Standardwerk von Norbert Elias erinnert Landweer, wie das Essen mit den Händen schambehaftet wurde. »Diejenigen Kreise, die Vorbild für andere sind, erwecken durch viele verschiedene Instanzen und Institutionen hindurch allmählich in anderen Kreisen die Scham, einen solchen Anblick zu bieten. [...] In der Folge sozialer Differenzierung werden Verhaltensweisen und Konventionen, die 
verliert allerdings ihre justierende und gemeinschaftbildende Kraft, wenn Anhänger einer immunisierten Kultur - gleichgültig ob religiösen oder politischen Inhalts - sich diesen Schamprozessen sehr grundsätzlich entziehen.

Spannend ist die Frage, ob wir im Prozess der Zivilisation aktuell in einer Phase sind, die verstärkt die Scham zur Peinlichkeit verdünnt.

\section{Archive der Scham und Peinlichkeit}

Ulrich Greiner, ehemals Feuilleton-Redakteur der ZEIT, hat eine beeindruckende kulturwissenschaftliche Studie über die Scham vorgelegt und sehr zurecht die Literatur gefeiert:

"Die Literatur ist ein hervorragendes Archiv, das die Wandlungen der Gefühlskultur sammelt und aufbewahrt. Der Komplex aus Schuld und Scham und Peinlichkeit zählt zu den stärksten Antriebskräften, die Literatur entstehen lassen: als Ausdruck eines unlösbaren Konflikts, als rückwirkende Schambewältigung, als Erklärungsversuch des Unverstandenen, vielleicht gar Unerklärbaren. ${ }^{15}$

Greiner legt »eine strukturelle Betrachtung« vor und verfolgt die These, "dass an die Stelle der alten Schuldkultur und der noch

bisher auf bestimmte Kreise oder Stände begrenzt waren, auf weitere Schichten bezogen. Über die latente Drohung von Ausgrenzung und sozialem Ausschluss und der entsprechenden Angst vor möglicher Beschämung können in einer Atmosphäre von Normenunsicherheit neue Normen etabliert werden, die in der jeweils nachfolgenden Generation fester im Gefühl verankert werden. «Das ist der entscheidende Punkt: Durch die Beschämung werden Normen im Leib verankert. Hilge Landweer, Scham und Macht, 163. Norbert Elias, Über den Prozeß der Zivilisation. Soziogenetische und psychogenetische Untersuchungen. Zweiter Band:Wandlungen der Gesellschaft. Entwurf zu einer Theorie der Zivilisation [1939], Frankfurt a.M. 1997, hier 358, 408. "[E]s ist diese als Selbstzwang angezüchtete Furcht vor der Verringerung des eigenen Ansehens in den Augen anderer, mag sie nun die Gestalt der Scham oder etwa die des Ehrgefühls annehmen. [...] [D]er Konflikt, der sich in Scham-Angst äußert, ist nicht nur ein Konflikt des Individuums mit der herrschenden, gesellschaftlichen Meinung, sondern ein Konflikt, in den sein Verhalten das Individuum mit dem Teil seines Selbst gebracht hat, der diese gesellschaftliche Meinung repräsentiert: es ist ein Konflikt des eigenen Seelenhaushalts; er selbst erkennt sich als unterlegen an. Er fürchtet den Verlust der Liebe oder Achtung von Anderen, an deren Liebe und Achtung ihm liegt oder gelegen war. Deren Haltung hat sich in ihm zu einer Haltung verfestigt, die er automatisch sich selbst gegenüber einnimmt."

${ }^{15}$ Ulrich Greiner, Schamverlust. Vom Wandel der Gefühlskultur, Reinbek bei Hamburg 2014, 21f. 
älteren Schamkultur eine neue Kultur getreten ist: die Kultur der Peinlichkeit ${ }^{16}$.

Angespielt wird von Greiner hier auf die Thesen, die vom Altphilologen Eric R. Dodds und dann von der Anthropologin Ruth Benedict ${ }^{17}$ in den 40er und 50er Jahren des 20. Jahrhunderts aufgestellt wurden und die für eine Unterscheidung zwischen Schamkulturen und Schuldkulturen plädierten: Die älteren Schamkulturen wurden geographisch im antiken Griechenland, im Orient und vor allem in Japan auch noch zu Zeiten des Zweiten Weltkriegs verortet; für die Schuldkultur stand exemplarisch das christliche Europa und das christliche Amerika Pate. Ob es eine temporale Logik und eine geographische Aufteilung derVerhaltenskulturen gibt, ist zwar zurecht bezweifelt worden, aber die Unterscheidung von Scham und Schuld hat eine bleibende Bedeutung, weil, wie oben angedeutet, nur im Rekurs auf Schuld keine Moralität aufgebaut werden kann. Noch problematischer für ethische Diskurse wäre die Ausdünnung der Scham zur Peinlichkeit. Mit der Brille von Hermann Schmitz gelesen: Wenn die Peinlichkeit ins Zentrum rückt, dann fehlt der moralische Ernst.

In seinen Vorlesungen über Ethik hat der Philosoph Ernst Tugendhat, der vielleicht prominenteste Vertreter einer autonomen Begründung der Ethik, noch einmal eine Unterscheidung angebracht, in welchen Settings sich Schamzeugen auch lustig machen dürfen, also nicht mit absolutem Ernst reagieren müssen:

"Scham ist das Gefuihl des Selbstwertverlustes in den Augen der möglichen anderen. Besonders scharf empfinden wir Scham, wenn andere wirklich anwesend sind und wenn wir sie als kompetent ansehen, z.B. ein Violinspieler, wenn er im Konzert schlecht spielt. Aber auch wenn er alleine übt, wird er sich, wenn er schlecht spielt, schämen - angesichts der Augen bzw. Ohren eines möglichen Publikums. Im Unterschied nun zu den speziellen Fähigkeiten, die wir im allgemeinen nur rudimentär ausbilden müssen, und gut nur, wenn wir unser Selbstwertgefühl mit ihnen verbinden, gibt es eine Fähigkeit, die für die Sozialisation zentral ist, und das ist die Fähigkeit, ein sozial umgängliches, ein kooperatives Wesen zu sein. [...] [I]ch möchte nun behaupten, daß die moralischen Normen einer Gesellschaft eben jene sind, die diese Standards festlegen, das heißt die definieren, was es heißt, ein kooperatives Wesen zu sein. In Urteilen, in denen

\footnotetext{
${ }^{16}$ Ebd., 24.

${ }^{17}$ Eric R. Dodds, Die Griechen und das Irrationale (1951), Darmstadt 1970; Ruth Benedict, Chrysantheme und Schwert: Formen der japanischen Kultur (1964), Frankfurt a.M. 2006.
} 
wir über Menschen und ihre Handlungen sagen, sie seien gut oder schlecht, beurteilen wir die Menschen nicht hinsichtlich spezieller Fähigkeiten, sondern im Hinblick auf diese zentrale Fähigkeit. Mit dieser Auffassung stimmt gut überein, daß wir uns nicht nur schämen können, wenn wir in einer bestimmten, uns wichtigen Fähigkeit versagen, sondern Scham ist auch die emotionale Reaktion, wenn wir moralisch versagen (also angesichts der Normen, die aus der Perspektive des Betreffenden sein Gutsein als kooperatives Wesen definieren). ${ }^{18}$

Dieser Unterschied manifestiert sich auch in der Reaktion der Schamzeugen: Im nichtmoralischen Fall kann das Publikum sich über den sich Schämenden lustig machen; im moralischen Fall wird der Beschämte mit dem Affekt der Empörung und mit Tadel rechnen müssen, weil es "um die gemeinsame normative Basis geht ${ }^{19}$. Die Gesellschaft kann von niemandem fordern, ein guter Geiger zu sein, aber sehr wohl ein kooperatives Wesen zu sein; wer dagegen verstößt, muss mit Sanktionen rechnen. Allerdings gilt:

"Für die bestimmte Sanktion der Empörung ist nur empfindlich, wer sie in der Scham internalisiert hat $[\ldots]$ und sich zu einer Totalität von Personen zugehörig versteht, die mittels der inneren Sanktion von Empörung und Scham wechselseitig voneinander fordern, die diese Identität ausmachenden Normen nicht zu verletzen. $\|^{20}$

Hellsichtig und für mich wegweisend kann Tugendhat sein Verständnis, die Moral an Schamerfahrungen rückzubinden, an Jesus von Nazaret verdeutlichen.

"Jesus hat die damals vorgegebene Moral seines Volkes relativiert und ergänzt. [...] Was der Reformator sagt, ist: Inhalte, über die ihr euch empört und schämt, sind nicht diejenigen, die dieser Gefühle würdig sind; die neuen Inhalte, die ich fordere, sind diejenigen, deren Einhaltung ihr voneinander wechselseitig fordern solltet. ${ }^{21}$

${ }^{18}$ Ernst Tugendhat, Vorlesungen über Ethik, Frankfurt a.M. 1993, 57 .

${ }^{19}$ Ebd., 58.

${ }^{20}$ Ebd., 60.

${ }^{21}$ Ebd., 63. 


\section{Der fehlende Scham-Blick in den Medien}

Greiner bleibt die Begründung für die nur nebenhin behauptete These, die Kultur der Peinlichkeit löse die Scham- und Schuldkulturen aktuell ab, schuldig. Die mögliche Antwort liegt in der konstanten Mediennutzung der Nerds und dem dadurch ausgelösten Fließprozess vom intimen Selbstbild zum immerwährenden Öffentlichkeitsbild. Weil Menschen in ihrer Lust (manchmal auch Sucht) sich auszudrücken, unentwegt Selfies posten, jedem Scoop hinterher rennen und die mediale Öffentlichkeit an bisher zumeist in privaten Innenräumen angesiedelten Gefühlsregungen teilhaben lässt, wird jede private Person zu einer teilweise öffentlichen Person, die immer mit Anfeindungen aus schlecht ausgeleuchteten Ecken rechnen muss und diese Gefahr einkalkuliert. Die Social media können auch zum digitalen Pranger werden. Der Shitstorm bricht dann über einem Mediennutzer zusammen. Aber offenbar wird diese Gefahr bewusst in Kauf genommen, um in der knallharten Ökonomie der Aufmerksamkeit ${ }^{22}$ zu reüssieren.

Auslöser für eine veritable Schamsituation ist ein persönlicher Blick (das kann selbstredend auch ein internalisierter Blick sein); ich verliere dann in einem Augenblick mein symbolisches Kapital im Auge eines (oder mehrerer) Personen, die mir viel bedeuten. Das Leben im Netz dagegen zielt auf eine Gruppe, die nach den Rändern hin diffundiert.

"Dadurch, dass immer mehr Menschen zu öffentlichen Menschen werden, verschiebt sich also die Funktion der Scham. Immer mehr Menschen müssen als öffentliche Menschen einen medialen Habitus entwickeln, der es erlaubt, öffentliche Entblößung nicht als Scham zu erleben. [...] Die das Internet als dominantes Medium verwendenden Menschen sind bereits öffentlich bloßgestellt und haben einen diesen Umstand angemessenen Habitus entwickelt - die öffentliche Existenz ist ihr

\footnotetext{
${ }^{22}$ So lautet ein viel diskutiertes Buch von Georg Franck, München ${ }^{10} 1998$. Im Anschluss an ein Buch von Bernhard Waldenfels klagt Johanna Haberer als Leitbegriff einer Medienethik >Aufmerksamkeit< ein: "Aufmerksamkeit hat immer eine soziale Konnotation. Aufmerksamkeit führt zur Beachtung. Beachtung, die wir schenken, kann zu Achtsamkeit führen, zu Rücksicht und Respekt." Johanna Haberer, Medienethik und die Rolle der christlichen Publizistik in der heutigen Medienlandschaft, in: ZEE 58 (2014), 261-272, hier 268. Haberer plädiert zudem (276-270) für Multiperspektivität, Befähigung und Beteiligung, für ein Wächteramt, fordert Medien als Stimme der Stimmlosen und als Instrumente der Freiheit.
} 
Normalfall. Eine öffentliche Bloßstellung wird unter diesen

Bedingungen nicht mehr als schamhaft erlebt. ${ }^{23}$

Vergleichbar öffentlichen Personen wie etwa Politikern, legt sich jeder Mediennutzer vorab eine Teflonhaut zu. Beinahe gar nichts ist diesen - nach der harten Währung der Aufmerksamkeit strebenden - Personen peinlich, weil sie in der Bildschirmpräsenz vor Scham-Blicken geschützt sind. Das korrelierende Fremdschämen ${ }^{24}$ oder besser: das korrelierende Gefühl der Peinlichkeit auf Seiten der Zuschauer ist prompt auch nur eine Schwundstufe der Scham, die $\mathrm{zu}$ schamlosen und stillosen Kommentaren herausfordert.

Von hier aus wird verständlich, warum Theologen wie etwa Wilfried Härle sehr nachdrücklich auf die Wichtigkeit direkter Begegnung abheben: "Die eigentliche sozialethische (und pädagogische) Herausforderung beginnt freilich [...] mit der Frage, wodurch Menschen die Lust an der direkten Kommunikation mit ihresgleichen geweckt werden kann. $\aleph^{25}$ Und Manfred Pirner erinnert:

"Von der theologischen Anthropologie her ist [...] der sozialleibliche Aspekt des Menschen als notwendige Ergänzung und Korrektur des medial-imaginativen einzubringen: Entsprechend dem anthropologischen Grundbedürfnis nach körperlicher und psychischer Zuwendung rrealisiert bzw. sverifiziert sich das medial vermittelte symbolische Universum christlichen Glaubens durch >leibhaftige< Nächstenliebe. ${ }^{26}$

Wie sind die anthropologischen Konsequenzen zu bewerten, die durch den fehlenden Schamblick in den Medienwelten ausgelöst werden? Wird dadurch ein gigantisches Programm der De-Sensibilisierung in Kraft gesetzt? Wird jede mögliche Schamerfahrung auf Peinlichkeit reduziert, die man weglachen kann? Oder wird, das

${ }^{23}$ Christian Swertz/Elsbeth Wallnöfer, Internet und Scham - Sensationen, Skurrilitäten und Tabus im Internet, in: Sensation, Skurrilität und Tabus in den Medien, hg. v. Sonja Ganguin/Uwe Sanders, Wiesbaden 2006, $75 f$.

${ }^{24}$ Das Fremdschämen, ein Begriff relativ jungen Datums, speichert die mediale Ausweitung des homo medialis. Ich halte den Begriff für ungenau, weil die Situation, die er einfangen soll, häufig ohne Ernst auskommt und besser durch den Begriff der Peinlichkeit eingefangen wird.

${ }^{25}$ Wilfried Härle, Sozialethik in der Mediengesellschaft, in: Medienethik. Freiheit und Verantwortung. Festschrift zum 65. Geburtstag von Manfred Kock, hg. v. Christian Drägert/Nikolaus Schneider, Christliche Publizistik Bd. 1, Stuttgart/Zürich 2001, 419-435, hier 434.

${ }^{26}$ Manfred L. Pirner, Extra media nullus homo? Theologische Aspekte zu einer Anthropologie der Medien, in: Matthias Rath/Manfred L. Pirner (Hg.), Homo medialis. Perspektiven und Probleme einer Anthropologie der Medien, München 2003, 113. 
wäre die positive Lesart, die Scham künftig reserviert für Fälle, wo das "Gutsein als kooperatives Wesen" (Tugendhat) auf dem Spiel steht? Nochmals: Wenn die oben zitierten Autoren recht haben und Schamerfahrungen für die Ausbildung von Sensibilität und Charakterformung, also für die Ausbildung eines kooperativen und situationssensiblen Habitus, entscheidend sind, würde eine Kultur der Peinlichkeit, die sich von der Scham-Kultur gelöst hat, den Raum der Moral erst gar nicht betreten. ${ }^{27}$ Ich bin optimistisch, dass dieser Loslösungsprozess nicht geschieht.

\section{Retrospektive Scham}

Kehren wir abschließend zu Robert Kisch zurück. Hier sind es Alltagserfahrungen, die ihn zu einer Neujustierung der Bewertung des früheren Lebens bewegen.

"Ja, früher, in meinem vorigen Leben, hätte ich wahrscheinlich wieder an einem \Lob der Marktwirtschaft gedichtet. So was in der Art habe ich früher gerne geschrieben. Kapitalismus ist cool, könnte fast aus Tempo stammen. Zumindest war es unser Denken.

Gewerkschaftsarbeit, o Gott, alleine der Ausdruck, das waren bärtige Zausel mit Plastiktüten auf dem Kopf. Blödsinnige Parolen auf stumpfsinnigen Demos, Trillerpfeifen und leeres Gelaber. Und ich war ein aufstrebendes Genie mit Lebensplänen. Vielleicht mal ein Jahr nach New York gehen?

Heute stecke ich mittendrin. Und kann nur noch krächzen, kein Loblied mehr.

Mag sein, dass die Marktwirtschaft als Konstrukt Innovationen ermöglicht - und nur die Marktwirtschaft Veränderungen durchsetzt -, aber ich stehe mitten im Orkan, unten, und mag nicht mehr singen.

Heute schreibe ich auch keine feuilletonistischen Feinsinnigkeiten mehr.

Früher...

Eine ekelige Welt, für die ich mich heute oft schäme.

Ich wusste nicht, was viele Menschen täglich durchleben müssen. $\aleph^{28}$

\footnotetext{
${ }^{27}$ Klaas Huizing, Scham und Ehre. Eine theologische Ethik, Gütersloh 2016.

${ }^{28}$ Kisch, Möbelhaus, $280 f$.
} 
Peinlichkeit hat sich zur retrospektiven Scham weiter entwickelt. Der Mann besitzt keine Teflonhaut, hat vielmehr die gegenwärtige Lebenserfahrung, auf die er gerne verzichtet hätte, verarbeitet. Er ist, um ein altes Wort zu gebrauchen, weise geworden. Ein Weiser ist selten peinlich.

- Klaas Huizing, Niederländer, hat einen Lehrstuhl für Systematische Theologie in Würzburg. Er veröffentlichte bisher 12 Romane und 13 Monographien. 2016 erscheinen Scham und Ehre. Eine Evangelische Ethik und Zu Dritt. Ein Barth-Roman. 